\title{
Eficácia de diferentes processos de desinfecção em turbinas de alta rotação
}

\section{Effectiveness of different disinfection processes in high speed turbines}

\author{
Igor Alcântara ${ }^{1}\left(\mathbb{D}\right.$, Renato Daniel de Freitas ${ }^{1}\left(\mathbb{D}\right.$, Ludimila Gomes Pinheiro ${ }^{2}\left(\mathbb{D}\right.$, Andrine Tavares Pereira Felipe ${ }^{3}(\mathbb{D}$, Pedro Diniz \\ Rebouças ${ }^{4}(\mathbb{D}$, Iriana Carla Junqueira Zanin dos Santo (i)
}

1. Discente do curso de Odontologia pela Universidade Federal do Ceará (UFC), Sobral, CE, Brasil. 2. Engenheira de Alimentos pela Universidade Federal do Ceará (UFC), Fortaleza, CE, Brasil. 3. Enfeira graduada pela Universidade Vale do Acaraú (UVA), Fortaleza, CE, Brasil. 4. Pós-graduando em Odontologia pela Universidade Federal do Ceará (UFC), Fortaleza, CE, Brasil. 5. Docente do curso de Odontologia pela Universidade Federal do Ceará (UFC), Sobral, CE, Brasil.

\section{Resumo}

Introdução: a contaminação cruzada pode ocorrer na prática odontológica, e a correta descontaminação das Turbinas de Alta Rotação (TAR) é um passo importante no controle desse tipo de infecção. Objetivo: determinar o protocolo de uso do detergente enzimático na limpeza das TARs que promova a maior redução microbiana da superfície do instrumento no menor tempo. Métodos: este estudo contou com uma amostra de 21 turbinas de alta rotação $(n=3) .0$ experimento foi dividido em cinco fases sequenciais: contaminação inicial, limpeza das turbinas, contagem microbiológica inicial, esterilização das turbinas e contagem microbiológica final. As amostras foram divididas em seis grupos: grupo (1): as turbinas foram envolvidas por 8 minutos em gaze embebida em detergente enzimático seguida por fricção mecânica; grupo (2): de forma semelhante ao grupo (1) pelo tempo de 4 minutos. No grupo (3), as turbinas foram, imediatamente, friccionadas com gaze embebida em detergente enzimático seguida de 8 minutos de espera, nos grupos (4) e (5), de forma semelhante ao grupo (3), pelo tempo de 4 minutos e zero minuto, respectivamente. No grupo (6), (controle negativo) não houve contaminação das TARs, e foram aguardados 8 minutos sem realização de nenhum protocolo de descontaminação, enquanto no grupo (7) (controle positivo), houve contaminação pelo pool salivar, mas nenhum protocolo de descontaminação foi realizado. Resultados: após segunda coleta, apenas o grupo (2) foi estatisticamente significante, mostrando-se como melhor protocolo de descontaminação das TARs. Conclusões: dessa forma, sugerimos a utilização de gaze embebida em detergente enzimático por 4 minutos, seguida de fricção mecânica com a gaze previamente ao procedimento de esterilização física por calor sob pressão, como protocolo de biossegurança.

Palavras-chave: Esterilização. Odontólogos. Bactérias.

\begin{abstract}
Introduction: cross contamination can occur the dental practice and the correct decontamination of high-speed turbines (HST) is an important step in the control of this type of infection. Objective: to determine the protocol of use of the enzymatic detergent in the cleaning of the TARs that promotes a greater microbial reduction of the surface of the instrument in the shortest time. Methods: This study had a sample of 21 high-speed turbines (n=3). The experiment was divided into 5 sequential phases (initial contamination, turbine cleaning, initial microbiological counting, turbine sterilization and final microbiological counting). The samples were divided into six groups: group (1) the turbines were wrapped for 8 minutes in gauze soaked in enzymatic detergent followed by mechanical friction, group (2) in a similar way to group (1) in the time of 4 minutes. In group (3) the turbines were immediately frictioned with gauze soaked in enzymatic detergent followed by 8 minutes of waiting, in groups (4) and (5) similarly to group (3) for the time of 4 minutes and zero minutes, respectively. In group (6) (negative control) there was no contamination of the HST and 8 minutes were awaited without any decontamination protocol, whereas in the group (7) (positive control) there was contamination by the salivary pool, but no decontamination protocol was performed. Results: After second collection, only group (2) was statistically significant, showing itself as the best decontamination protocol for HST. Conclusions: we suggest the use of gauze soaked in enzymatic detergent for 4 minutes followed by mechanical friction with the gauze prior to the procedure of physical sterilization by heat under pressure as a biosafety protocol.
\end{abstract}

Key words: Sterilization. Dentists. Bacteria.

INTRODUÇÃO

A prática odontológica apresenta uma série de peculiaridades que constituem fator de risco para a ocorrência de contaminação cruzada, seja entre pacientes, seja entre paciente e equipe de saúde bucal. Entre esses fatores, pode-se citar o contato direto dos instrumentos e profissionais com fluidos corporais, como saliva e sangue; a formação de aerossóis durante a utilização da turbina de alta rotação ou da seringa tríplice e também devido ao fato de muitos instrumentos serem pontiagudos e perfuro cortantes ${ }^{1}$.

Inegavelmente, os instrumentos odontológicos são potenciais fontes de contaminação e disseminação microbiana entre pacientes, ao albergar e carrear um universo de microrganismos, o que justifica o gerenciamento seguro em seu processamento ${ }^{1,}$ 2, 3, 4. Esse processamento deve ser realizado a fim de eliminar todas as formas de vida microbiana da superfície dos instrumentos, tornando-os seguros para serem utilizados nos pacientes, sem, no entanto, danificar os instrumentos ${ }^{1}$.

Entretanto, segundo Pinto, 2013 uma lacuna nos conhecimentos atuais em controle de infecção cruzada na prática odontológica relacionada a produtos refere-se à discussão de métodos e 
técnicas para a descontaminação das Turbinas de Alta Rotação $(T A R)^{5}$. Esses equipamentos consistem em produtos de alto custo que possuem sistemas de rolamento interno. Esses fatores contribuem para que o processo de descontaminação das TARs seja, ainda, um desafio, uma vez que deve associar-se à segurança da descontaminação e à manutenção da integridade física do equipamento.

As TARs são utilizadas em diversos tipos de procedimentos na prática odontológica, que apresentam diversos graus de complexidade e de contato com material contaminante, como preparo de cavidades para restaurações, acesso coronário, osteotomias e odontosecções. Pelos diversos procedimentos e de acordo com o objetivo de seu uso, a TAR é classificada como um material crítico ou semicrítico ${ }^{5}$.

Dentro da classificação de risco em causar infecção, a recomendação oficial pelas sociedades científicas para descontaminação segura da TAR consiste na limpeza prévia com água, detergente e friç̧ão mecânica, e posterior esterilização por autoclavagem ${ }^{6,7}$. A realização da limpeza prévia é um quesito primordial para a obtenção de uma completa esterilização do instrumental, uma vez que o vapor sob pressão só é capaz de esterilizar superfícies, não sendo capaz de penetrar no interior de sujidades. Sendo assim, a limpeza deve ser realizada minuciosamente, garantindo a remoção da sujidade e contribuindo para a redução da contaminação microbiana ${ }^{7}$.

Dessa forma, o objetivo deste trabalho é determinar o protocolo de uso do detergente enzimático na limpeza das TARs que promova a maior redução microbiana da superfície do instrumento no menor tempo.

\section{MÉTODOS}

Este estudo contou com uma amostra de 30 turbinas de alta rotação ( $n=5$ por grupo). Todas as canetas utilizadas foram, previamente, processadas na Central de Material Esterilizado (CME) do Curso de Odontologia da Universidade Federal do Ceará - Campus Sobral e entraram no experimento esterilizadas por método físico de vapor sob pressão em autoclave, seguindo todas as normativas de biossegurança da Agência Nacional de Vigilância Sanitária - ANVISA․

Para assegurar o mesmo nível de contaminação microbiana das amostras, um pool de saliva humana foi utilizado para contaminar todas as canetas de maneira idêntica. Para tanto, $20 \mathrm{ml}$ de saliva humana foram coletados de três voluntários saudáveis, em jejum e com supressão de higiene oral por $12 \mathrm{~h}$, totalizando um volume de $60 \mathrm{ml}$ de saliva coletada. Para a coleta, o fluxo salivar foi estimulado com a mastigação de um filme plástico (Parafilm $\mathrm{M}^{\circledR}$ Pechiney Plastic Packaging, Akron, Ohio, EUA), a saliva produzida durante o primeiro minuto foi deglutida para, então, ser coletada em tubos tipo Falcon de 50 $\mathrm{ml}$ estéreis até o volume individual de $20 \mathrm{ml}$. A seguir, as salivas dos três voluntários foram misturadas para formar um pool de saliva humana. A essa amostra foram adicionados $60 \mathrm{ml}$ de tampão de adsorção ( $\mathrm{KCl} 50 \mathrm{mM}, \mathrm{KPO} 41,0 \mathrm{mM}, \mathrm{CaCl} 22 \mathrm{H} 2 \mathrm{O}$ $1,0 \mathrm{mM}, \mathrm{MgCl} 26 \mathrm{H} 2 \mathrm{O} 0,1 \mathrm{mM}, \mathrm{pH}=6,5)$ para assegurar a sua preservação, e o material foi mantido em gelo até o momento da contaminação das canetas.

O experimento foi dividido em 5 fases sequenciais conforme descrito abaixo

Figura 1. Fases sequencias do experimento

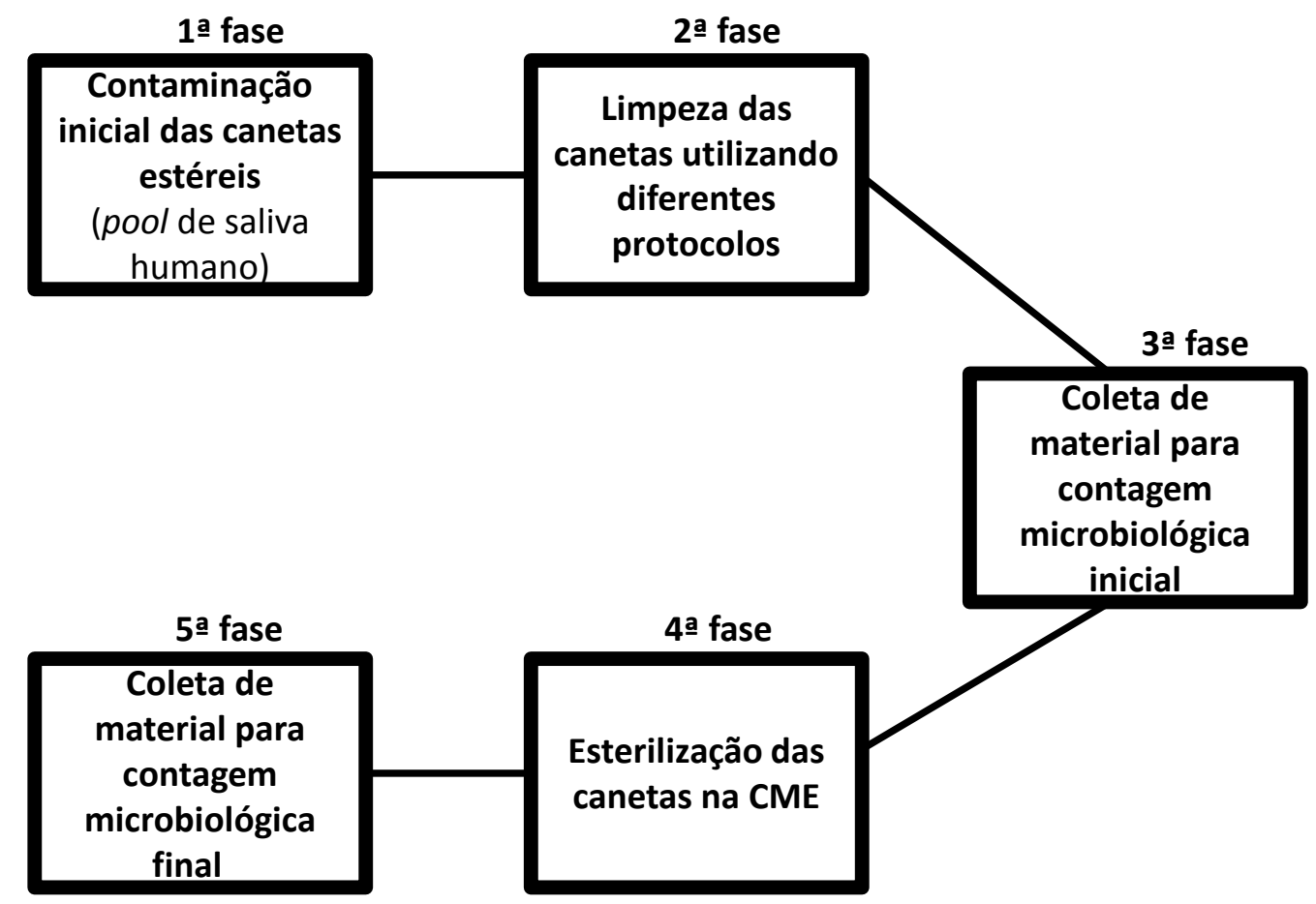


Contaminação inicial das canetas estéreis com pool de saliva humana

Sobre um campo estéril em câmera de fluxo laminar (TROX ${ }^{\circledR}$ TECHNIK, série 2030, Curitiba, Brasil), o operador realizou a abertura do pacote de papel grau cirúrgico estéril vindo da CME de maneira asséptica para prevenir contaminação da amostra. Sempre que havia a necessidade de repousar a amostra sobre a bancada, ela era mantida sobre a face interna do lado plástico do papel grau cirúrgico.

A contaminação foi limitada à metade superior da turbina, sendo delimitada pelo código de impressão existente na turbina de alta rotação, ficando a parte inferior para que o operador realizasse a sua apreensão. Para a realização da contaminação, a TAR foi dividida em cinco partes, sendo quatro localizadas representativas de cada uma das quatro faces da turbina, e a quinta parte representada pela cabeça da turbina. Para a contaminação das amostras, o swab estéril (ABSORVE ${ }^{\circledR}$, Jlangsu Rongye Technology, China) foi emergido uma única vez no pool de saliva humana, retirado e friccionado com quatro movimentos repetitivos em cada uma das cinco partes da caneta, a fim de garantir a contaminação uniforme da amostra. Após realização da cominação de todas as faces, o swab foi descartado, e a amostra passou para a fase de limpeza das turbinas de alta rotação, seguindo diferentes protocolos de desinfecção.

\section{Limpeza das canetas utilizando diferentes protocolos}

A amostra foi dividida, igualmente, em seis grupos com cinco turbinas de alta rotação em cada um deles $(n=5)$. Cada grupo representou uma técnica de limpeza das turbinas, utilizando solução de detergente enzimático (PODEROSO DETERGENTE MULTIENZIMÁTICO ${ }^{\circledR}$, Kelldrin, Anápolis, Goiás) empregado em tempo e condições distintas, sendo eles:

GRUPO 1 (8min EM): turbina envolvida em gaze embebida em detergente enzimático por 8 minutos, seguida por fricção mecânica com a gaze.

GRUPO 2 (4min EM): turbina envolvida em gaze embebida em detergente enzimático por 4 minutos, seguida por fricção mecânica com a gaze.

GRUPO 3 (8min ES): turbina imediatamente friccionada com gaze embebida em detergente enzimático seguida de 8 minutos de espera sem a gaze.

GRUPO 4 (4min ES): turbina imediatamente friccionada com gaze embebida em detergente enzimático seguida de 4 minutos de espera sem a gaze.

GRUPO 5 (0min ES): turbina foi imediatamente friccionada com gaze embebida em detergente enzimático sem nenhum tempo de espera.

GRUPO 6 (CN): controle negativo - turbina não foi contaminada, aguardaram-se 8 minutos na bancada, sem que nenhum protocolo de descontaminação fosse realizado e, então, foi realizado enxague com água.

GRUPO 7 (CP): controle positivo - turbina foi contaminada pelo pool salivar aguardaram-se 8 minutos na bancada, sem que nenhum protocolo de descontaminação fosse realizado.

Após a limpeza das turbinas de alta rotação, utilizando os diferentes protocolos de descontaminação descritos, as turbinas foram repassadas para outro operador treinado, o qual realizou sua lavagem. Para isso, uma gaze estéril foi embebida em água e friccionada pelo corpo da turbina, assegurando a remoção de todo o detergente enzimático, conforme protocolo de enxágue descrito pelo fabricante. Vale destacar que os operadores apreenderam a caneta apenas pela extremidade inferior, para evitar a contaminação da parte da turbina em estudo.

\section{Coleta de material para contagem microbiológica inicial}

Após o enxágue, a amostra retornou para o fluxo laminar em que a coleta para análise microbiológica foi realizada. Para tanto, microtubos para centrífuga estéreis foram previamente preparados e identificados para cada amostra correspondente ao puro e às diluições 1:10, 1:100 e 1:1000. O tubo correspondente ao puro continha $1 \mathrm{ml}$ de solução de $\mathrm{NaCl} 0,89 \%$ estéril, e os demais continham $900 \mu \mathrm{l}$ da mesma solução a fim de permitir a realização das diluições seriadas. Dessa forma, um novo swab estéril foi emergido uma única vez no tubo puro, contendo $\mathrm{NaCl}$ 0,89\%, retirado e friccionado com quatro movimentos repetitivos em cada uma das cinco partes da caneta a fim de garantir a coleta uniforme da amostra. Ao final da coleta, o swab foi seccionado próximo à sua extremidade, dentro do microtubo identificado como puro. Realizou-se a agitação em vortex (VORTEX MIXER ${ }^{\circledR}$, Velp Scientifica, Usmate, Itália) por 10 segundos seguidos da diluição seriada. O plaqueamento foi realizado em triplicada, em placas de petri, contendo $60 \mathrm{ml}$ de meio de cultura de BHI Ágar - Brain Heart Infusion Ágar (Kasvi ${ }^{\circledR}$, Itália). As placas foram incubadas em aerobiose a 37으 por $48 \mathrm{~h}$. Em seguida, o número de unidades formadoras de colônia por $\mathrm{ml}$ de amostra (UFC/ml) foi estabelecido.

\section{Esterilização das canetas na CME}

Após a coleta ter sido realizada em toda a superfície estudada da caneta, elas foram secas com gaze estéril e foram reembaladas em papel grau cirúrgico identificado com o grupo do estudo ao qual pertenciam e foram esterilizados em autoclave (PHOENIX ${ }^{\circledR}$ LUFERCO, Araraquara, Brasil) a 121 ㅇ C por 15 minutos, segundo protocolo utilizado na CME. Após a esterilização, as canetas retornaram ao laboratório e foram submetidas à nova coleta para contagem microbiológica, conforme descrito no item 2.3.

\section{Análise estatística}

Os dados foram obtidos e tabulados no programa Excel 2013 e submetidos à análise descritiva. Para a análise estatística, foi utilizado o programa BioEstat 5.0 (Mamirauá, Pará, Brasil), em que a normalidade dos dados foi avaliada pelo teste de Kolmogorov-Smirnov. O teste de Análise da Variância (one-way ANOVA) com post-hoc de Bonferroni, considerando um nível de significância de 5\%, foi utilizado. 


\section{RESULTADOS}

Os resultados do estudo podem ser observados no figura 2. Foi notória a maior eficácia dos processos que utilizaram a associação de envolver a turbina de alta rotação em gaze embebida com detergente enzimático, seguido pela fricção mecânica com gaze, sendo esses processos decisivos para menor contagem de UFCmL-1. Após a segunda coleta, todos os grupos apresentaram diminuição da quantidade de UFC/ $\mathrm{mL}$ quando comparado à contagem inicial. A comparação dos tratamentos evidenciou que apenas o grupo utilizando turbina envolvida em gaze, embebida em detergente enzimático por 4 minutos, seguida por fricção mecânica com a gaze, diferiu, estatisticamente, dos demais nas duas coletas microbiológicas realizadas, se mostrando como melhor protocolo de descontaminação das turbinas de alta rotação entre os testados.

Figura 2. Resultados em UFC/mL de primeira e segunda coleta após descontaminação das turbinas de alta rotação (TAR).

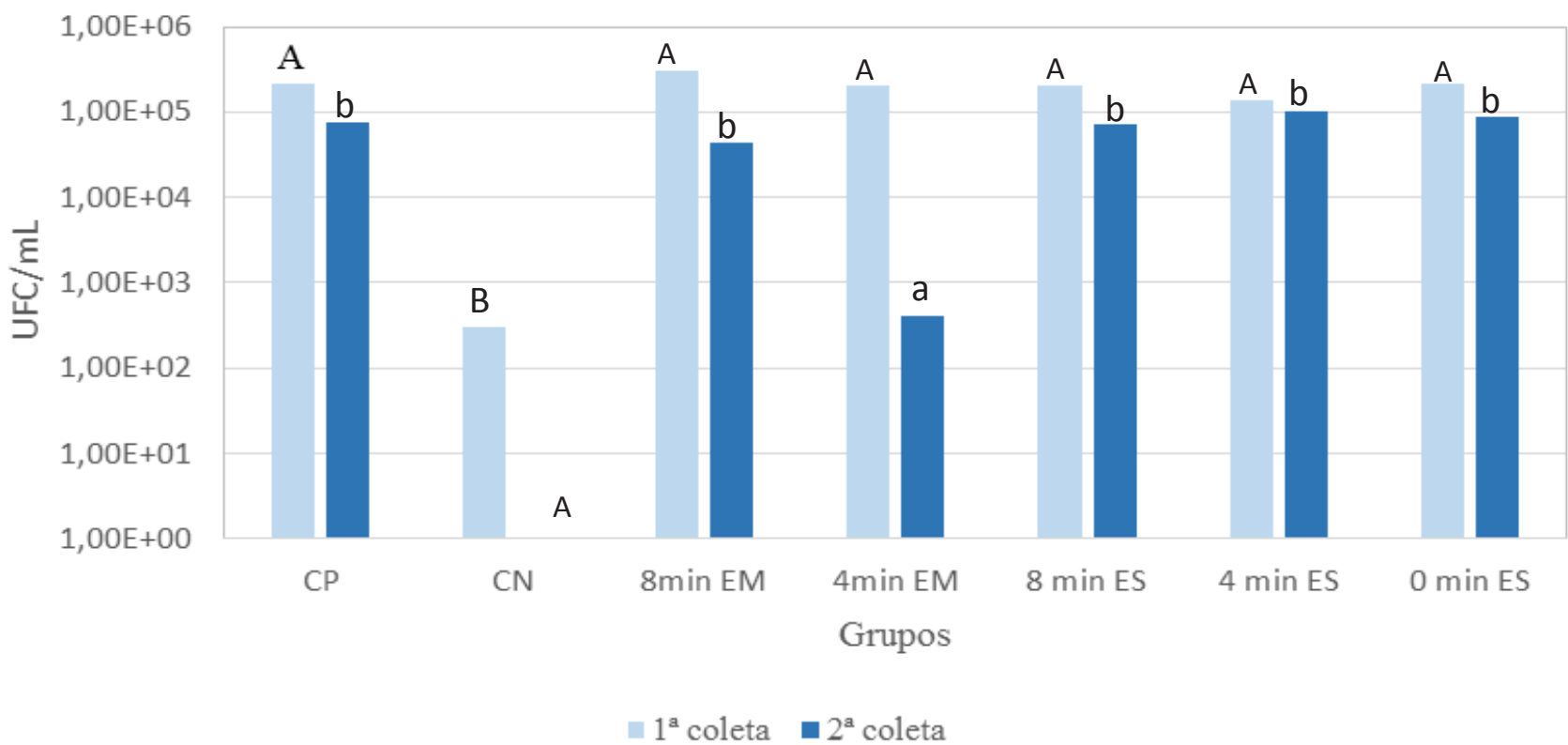

Médias das contagens microbiológicas em UFC/ml da primeira coleta (após limpeza) e da segunda coleta (após limpeza seguida de esterilização em autoclave) das turbinas de alta rotação. Letras maiúsculas comparam os resultados obtidos nos diferentes tratamentos na primeira coleta, e letras minúsculas comparam os resultados obtidos na segunda coleta $(n=3)$.

\section{DISCUSSÃO}

A necessidade deste estudo surgiu da dúvida do melhor, mais eficiente e mais rápido protocolo para descontaminação das turbinas de alta rotação por professores e alunos do curso de Odontologia da Universidade Federal do Ceará - Campus Sobral e foi realizado a fim de estabelecer o protocolo de descontaminação das turbinas de alta rotação a ser adotado no curso.

É de suma importância definir que essas coletas tiveram por objetivo esclarecer a necessidade do protocolo que melhor leva à desinfecção. Se todos ficassem em condições de uso após a esterilização, poderíamos optar pelo protocolo menos complicado e mais rápido; mas, a partir da segunda coleta microbiológica, foi possível perceber, ainda, um residual de contaminação dos materiais analisados.

A cavidade bucal abriga mais de 700 micro-organismos diferentes em sua flora9, muitos dos quais podem contaminar pacientes e profissionais por transferência de saliva, de sangue, do fluido crevicular gengival e até das secreções respiratórias do paciente, com possibilidade de causar patologias que vão da tuberculose ao herpes, de um leve resfriado a uma pneumonia, atuando como meios efetivos de transmissão e infecção de doenças ${ }^{10}$. As peças de mão e as cadeiras odontológicas obtiveram um índice de maior contaminação, sendo mais prevalente nas TARs a ocorrência de cocos e bastonetes grampositivos e diplococos gram-negativos ${ }^{11}$.

A esterilização, há bastante tempo, vem sendo discutida, sempre preconizada com o máximo rigor, a fim de garantir que um artigo não crítico ou semicrítico não se torne crítico durante o procedimento por uma errada descontaminação e/ou esterilização prévia ${ }^{12}$. o protocolo de biossegurança inclui o uso de equipamentos de proteção individual, como máscaras descartáveis, óculos e luvas descartáveis, os quais são considerados eficientes e diminuem, significativamente, os níveis de contaminação ${ }^{13,14}$. Entre os processos de esterilização, o processo físico pode ser efetuado por meio de vapor saturado sob pressão e calor a seco ${ }^{15}$, sendo o processo por vapor saturado sob pressão aquele que oferece maior segurança e economia ${ }^{16}$. 
Uma limpeza prévia nos instrumentos antes da esterilização final é necessária e fundamental para garantir a efetividade do método de esterilização e total biossegurança, pois diminui a carga microbiana e as sujidades. Considerando que o método de vapor sob pressão só funciona sobre superfícies e que ele não tem ação em profundidade, é fundamental que crostas, sangue e outras substâncias sejam removidas em etapa prévia à esterilização, para que o vapor possa entrar em contato direto com a superfície do instrumental a ser esterilizado. Quanto mais bem feita a limpeza, haverá maior chance de eficácia no processo de esterilização, e, caso não haja uma limpeza adequada, haverá uma elevação nos números de insucessos em relação à eliminação de patógeno ao final do processo de esterilização ${ }^{17}$.

O detergente enzimático já demonstrou, previamente, sua eficácia em artigos, utilizando sangue e sujidades17. Os resultados de nosso estudo corroboram esses achados, apresentando a necessidade de ação inicial do detergente enzimático por 4 minutos, seguida de fricção mecânica da gaze como a melhor técnica de desinfecção das turbinas de alta rotação previamente à lavagem e à sua esterilização em autoclave, o que também vai de acordo com as instruções de uso do produto utilizado.

\section{REFERÊNCIAS}

1. Jorge AOC. Princípios de biossegurança em Odontologia. Rev Biocienc. 2002 Jan-Jun; 8(1): 7-17.

2. Rutala WA, Weber DJ, HICPAC Members. Guideline for disinfection and sterilization in healthcare facilities, 2008. EUA; 2017.

3. Rutala WA, Weber DJ. Sterilization, High-Level Disinfection, and Environmental Cleaning. Infect Dis Clin N Am. 2011 Mar; 25(1):45-76. doi: 10.1016/j. idc.2010.11.009.

4. Alvarenga CF, Reis C, Tipple AFV, Paiva EMM, Sasamato SAA. Efetividade de um protocolo de processamento na esterilização de canetas de alta rotação em autoclave gravitacional. Rev Eletr Enf. 2011Jul-Set;13(3):560-5.

5. Pinto FMG. Desinfecção das canetas de alta rotação com álcool $70 \% \mathrm{p} / \mathrm{v}$ sem limpeza prévia: avaliação dos riscos de infecção cruzada [tese]. São Paulo (SP): USP; 2013.

6. ADA Council on Access, Prevention and interprofessional Relations; ADA Council on Scientific Affairs. Using mouth guards to reduce the incidence and severity of sports-related oral injuries. J Amer Dent Associa, 2006 Dec;137(12): 1712-20.

7. CDC. Guidelines for infection control in dental health-care settings. USA, 2003. [acesso 2019 Jan 15]; Disponível em: https://www.cdc.gov/oralhealth/ infectioncontrol/pdf/safe-care-checklist.pdf.

9. Aas JA, Paster BJ, Stokes LN, Olsen I, Dewhirst FE. Defining the normal bacterial flora of the oral cavity. J Clin Microbiol. 2005 Nov; 43(11):5721-32.

10. Pereira RS, Tipple AFV, Reis C, Cavalcante FO, Belo TKAMC. Análise microbiológica de canetas odontológicas de alta rotação submetidas à descontaminação com álcool etílico a 70\%. ROBRAC. 2008;17(44):124-132.

11. Rodrigues KA, Lima IF, Silva AK, Sotte DMKS, Silva JS. Análise de contaminantes microbiológicos em consultório odontológico. Pens@r Acadêmico. 2018; 4(1)16.

12. Chinellato LEM, Scheidt W. A. Estudo e avaliação dos meios de biossegurança para o cirurgião-dentista e auxiliares contra doenças infecto-contagiosas no consultório odontológico. Revista da FOB. 1993;1(1/4):59-66.

13. Bulgarelli AF, Torquato TM, Costa LSS, Ferreira ZA. Avaliação das medidas de biossegurança no controle de infecção cruzada durante tratamento periodontal básico. RBO. 2001 Jan; 58(3):188-190.

14. Gonçalves ES, Godoy SAL, Tripodi J. Manual de Biossegurança. 2. ed. Bauru: USP; 2014 [acesso 2019 Jan 10]. Disponível em: http://www.youblisher.com/

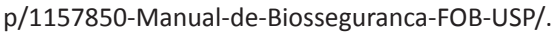

15. Biachi EC, Silva EJ, Cezar FAG, Aguiar PR, Bianchi ARR, Freitas CA, Riehl H. Aspectos Microscópicos da Influência dos Processos de Esterilização em Pontas Diamantadas. Mat. Res. 2003 Abr-Jun; 6(2):203-210. doi: http://dx.doi. org/10.1590/S1516-14392003000200015.

16. Gil RF, Camelo SH, Laus AM. Atividades do enfermeiro de centro de material e esterilização em instituições hospitalares. Acta Paul Enferm. 2007 Out-Dez; 20(4): 470-5.

17. Schmidt DRS, Yonekura CSI, Gil RF. Instrumento para avaliação de detergentes enzimáticos. Rev esc enferm USP. 2008; 42(2): 282-9. doi: http:// dx.doi.org/10.1590/S0080-62342008000200011. 